\title{
Design and Characterization of the Michigan Hypersonic Expansion Tube Facility (MHExT)
}

\author{
Yasin M. Abul-Huda*, Mirko Gamba ${ }^{\dagger}$, \\ The University of Michigan, Department of Aerospace Engineering Ann Arbor, MI 48104
}

\begin{abstract}
The development of the new hypersonic impulse facility at the University of Michigan capable of generating aerothermal flow conditions representative of flight Mach numbers ranging between 4 and 11 is discussed. Three conditions were designed and run extensively for the purposes of validating and characterizing the generated flows. The conditions were chosen to span a range of accessible flow properties while maintaining relevance to the studies of supersonic combustion. The performance of the facility was assessed based on its ability to repeatedly and accurately generate flow conditions of interest. The temporal and spatial uniformity of the flow were determined using simultaneous pitotstatic pressure measurements, pressure measurements with a pitot rack, and schlieren imaging. Flow uniformity, useful test time, and core-flow sizes are established. A shot-to-shot statistical analysis of the resulting shock speeds reveal their variability to amount to a fraction of a percent, attributing to the ability of the facility to consistently generate the desired flow conditions.
\end{abstract}

\section{Introduction}

$\mathrm{C}^{\mathrm{OR}}$ the past 50 years, the development of scramjet technology has relied heavily on ground test facilities for design validation purposes. The underlying physics present in the supersonic/hypersonic flow regime is of a highly complex and coupled nature in which the effects of chemistry, nonequilibrium, turbulence, and compressibility each play an important role in governing the behavior of the fluid flow. Understanding each of these aspects individually and how they affect one another is of key importance when designing the next generation of hypersonic aircraft.

Recently, there have been efforts to advance hypersonic flight technology through a number of flight experimentations using instrumented ballistic re-entry vehicles to study several hypersonic flow phenomena. Collaborative efforts such as the HIFiRE and HyShot programs have successfully tested sub-scale scramjet vehicles in true-flight environments [1-3]. Some of the objectives of these flights were to test the performance of dual-mode to scramjet-mode transition, demonstrate Mach flight numbers ranging between 6 and 8, the use of hydrocarbon fuels, laminar to turbulent boundary layer transition, and more. In spite of these successful missions, the utilization of ground test facilities remains the workhorse for the advancement of hypersonic technology due to its relatively simpler and lower cost of operation.

Impulse facilities such as shock tunnels, expansion tubes and expansion tunnels, allow the replication of high Mach number and high enthalpy flows. A number of these facilities have been constructed in the effort of studying supersonic flows present in several applications such as re-entry vehicles or scramjet combustors. Several of these facilities include the Large Energy National Shock Tunnel Facilities at CUBRC [4], NASA's HYPULSE facility [5], and the X-series facilities at the University of Queensland [6], the Hypervelocity Expansion Tube (HET) at CalTech [7], and the 6 inch expansion tube at Stanford [8].

The present work is aimed to describe the design and characterization of the newly developed Michigan Hypersonic Expansion Tube Facility (MHExT). Our system was designed to generate aeorthermal flow conditions representative of flight Mach numbers ranging between 4 and 11. However, the design was optimized to achieve maximum test time for conditions representative of Mach 6 - 8 flight to primarily study supersonic combustion phenomena. A total of three flow conditions were chosen for the work of characterizing the facility, where two of the flow conditions are representative of the combustor entry conditions for the recent HIFiRE II project.

\section{Background}

\section{A. Expansion Tube Theory}

The expansion tube operation was originally proposed and analyzed theoretically by Trimpi [9]. Similar to other impulse facilities, it is capable of generating high enthalpy and Mach number flows representative of conditions found

\footnotetext{
* Graduate Research Assistant, Department of Aerospace Engineering, AIAA Student Member, yas inma@umich . edu

$\dagger$ Assistant Professor, Department of Aerospace Engineering, AIAA Member, mirkog@umich . edu
} 


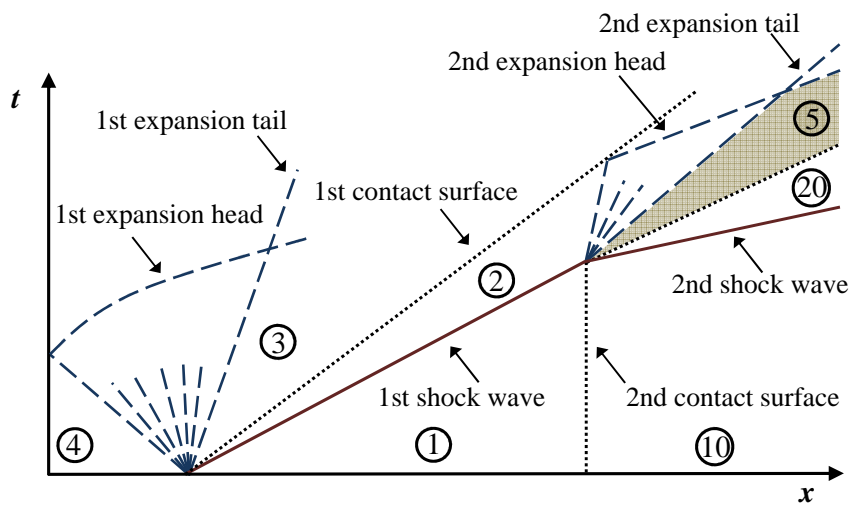

Figure 1. Schematic space-time diagram showing the wave processes present during the operation of an expansion tube. The numbers correspond to different regions or states of the various gases. Labeling of the states follows the original work by Trimpi [9].

in supersonic combustors and re-entry vehicles. An expansion tube is comprised of three main parts referred to as the driver, driven, and expansion sections. Before each test run, the sections are separated by diaphragms and filled with gases to predetermined pressures. The driver is the high pressure section which is filled with a gas with a high sound speed, typically helium. The driven section is filled with the test gas of interest at a low pressure, while the expansion section is also filled to a low pressure but typically with helium. In the simplest terms, an expansion tube can be thought of as two shock tubes being operated in tandem, where the first shock tube is comprised of the driver and driven sections of the expansion tube, and the second shock tube is comprised of the shock-processed driven section and the expansion section of the expansion tube.

What differentiates an expansion tube from other impulse facilities is the process used to reach the final aerothermodynamic state of the test gas. In particular, after being compressed by the incident shock wave generated by the rupture of the primary diaphragm, the test gas is further processed by an unsteady expansion wave generated by the rupture of the secondary diaphragm. This results in further acceleration of the test gas to higher Mach numbers as well as an increase in its total enthalpy. The process is shown in Fig. 1, which is a space-time diagram illustrating the wave processes that are present during the operation of an expansion tube. As shown, a second pair of waves is formed at the secondary diaphragm upon arrival of the incident shockwave. The second shockwave continues propagating through the expansion gas (region 10), while the expansion wave processes the test gas to its final state (region 5). Since the induced bulk motion of the test gas due to the incident shock wave is usually supersonic (region 2), the secondary expansion wave is swept downstream as shown in the figure. The test time begins with the arrival of the contact surface between regions 20 and 5, and is limited by the arrival of either of the three rarefraction waves, being the second expansion tail, the second expansion head reflected off of the first contact surface, or the first expansion head reflected off of the driver end wall.

There are a number of advantages that expansion tubes have over other impulse facilities such as reflected shock tunnels. First, expansion tubes can be operated over a wide range of conditions by only varying the types of gas, and initial fill pressures of each individual section. This eliminates the requirement of replacing a nozzle to alter the test gas Mach number. Secondly, the process never involves stagnating the test gas, resulting in flows with negligible amounts of dissociation and thermochemical non-equilibrium, and are thus more representative of the flight conditions of interest being replicated. This is imperative in high-speed combustion studies as the chemical composition of the test gas is a significant parameter that must be replicated accurately. Furthermore, higher stagnation pressures and temperatures are achieved in the test gas with an expansion tube for the same initial driver fill pressure and gas sound speed as a shock tunnel. This is accomplished by the unsteady expansion that processes the test gas after the rupture of the secondary diaphragm.

Nevertheless, the advantages of using an expansion tube do come at a cost as they are inherently limited by nonideal effects such as flow disturbances and boundary layer growth. There are number of adverse effects that result from this, which include: non-uniformity in the test gas flow properties, shortened test times, and a decrease in the effective core flow size. The present work aims to characterize the flows generated by the facility, investigating the extent in which these non-idealities affect the flows. Therefore, the work will be used to define useful bounds such as core flow size, spacial and temporal flow uniformity, and useful test time for future supersonic combustion studies. 


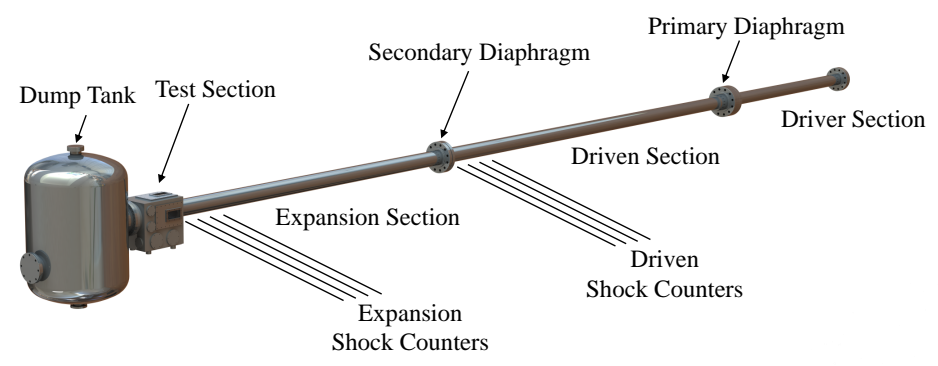

Figure 2. Schematic diagram of the MHExT Facility.

\section{B. Design and Operation Overview of MHExT}

The MHExT facility is composed of a $14 \mathrm{~m}$ long stainless steel pipe, comprised of the three main sections. A schematic of the completely assembled facility is shown in Fig. 2. The driver, driven, and expansion sections have a honed and polished internal diameter of about $144 \mathrm{~mm}$ and are $2.9 \mathrm{~m}, 8 \mathrm{~m}, 3 \mathrm{~m}$ long respectively. The tube is comprised of seven subsections so that test times can be optimized by leveraging its modular design. The entire tube rests on rollers to allow for easy movement of the pipe for diaphragm changing as well as an easier way to interchange the subsections. The dump tank and driver section each rest on their own independent carriage structures. This allows for complete detachment in order to more easily access the interior of the tube for routine inspections and cleaning.

The driver and driven sections are separated by the primary diaphragm which employs a double diaphragm design. This configuration shown schematically in Fig. 3, uses an intermediate section referred to as the buffer to better control rupture initiation of the diaphragms, leading to an increase in repeatability of test conditions between individual runs. This is accomplished by isolating the buffer with one or several $1 \mathrm{~mm}$ thick polycarbonate sheets and filling it with an intermediate pressure relative to the driver and driven sections. Upon evacuating the buffer, the upstream diaphragm rapidly ruptures due to the increase in pressure differential across it and is shortly followed by the rupture of the downstream diaphragm. These sheets are typically pre-scored with a cross pattern such that upon rupture, the diaphragms open into four petals. The regions of the tube in which the petals open into are square in cross-section. This allows the wave processes and core flow to develop in the shortest amount of time while introducing the least amount of disturbances and giving way to a more uniform and useful test gas flow.

The driven and expansion sections are separated by thin mylar sheets, typically $2 \mu \mathrm{m}$ in thickness. Previous studies by Miller et al. [10] have shown that secondary diaphragm thickness has no measurable effect on the final test gas properties for a fixed condition of interest. However, using thinner membranes results in the reduction of noise measured in the test gas. Therefore, the secondary diaphragm's thickness was kept to a minimal value for all of the experiments presented in this work.

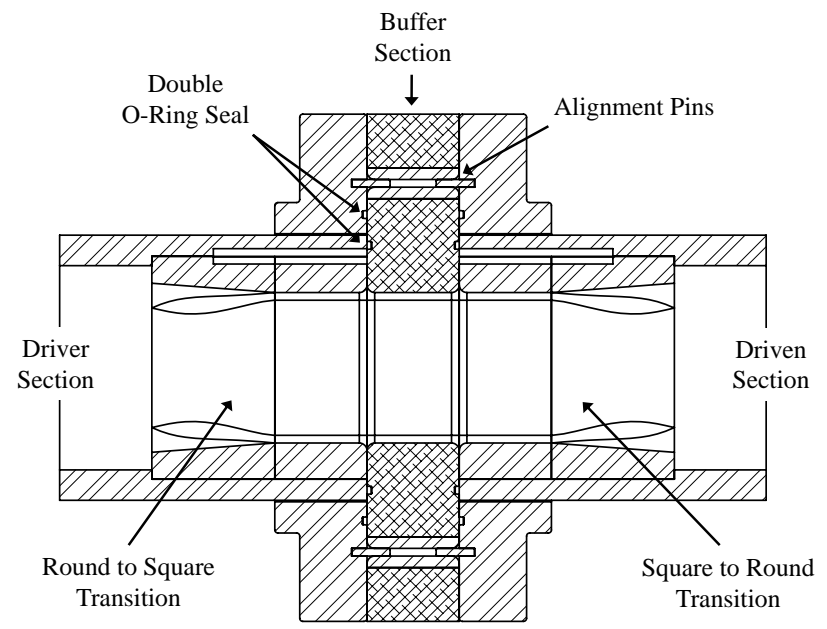

Figure 3. Cross-sectional schematic of the primary double-diaphragm, which uses an intermediate buffer section to control the initiation of a test. 
A test section and dump tank are located at the end of the expansion section and are mated with one another through a rubber expansion joint. This allows the dump tank to recoil relative to the test section during each test run, while maintaining the required sealing. The test section is a stainless steel rectangular chamber $45.7 \mathrm{~cm} \mathrm{x} 48.3 \mathrm{~cm}$ $\mathrm{x} 34.3 \mathrm{~cm}$ in size. Three quartz windows are used for optical access through the test section in order to carry out non-intrusive diagnostic methods. Several different-sized feed-through ports and access panels are distributed along its sides. The test section is independently anchored to the laboratory's floor and it is joined to the expansion section through dynamic o-rings to allow for relative movement of the tube while providing vacuum-tight sealing. The dump tank is approximately 1 cubic meter in volume and was designed to be sufficiently large such that properties in the test gas are constant during the usable test time.

Due to the inherently large convective speeds of the wave processes and test gas, data such as those from pressure transducers are sampled at a rate of $2 \mathrm{MHz}$. Data acquisition and control of the system is accomplished using a computer based National Instruments system. The NI system is comprised of a 9-Slot PXIe-1078 Chassis which houses two NI PXI-6133 DAQs, one NI PXI-2521 relay module, and a PXIe-PCIe8381 controller. The system is instrumented with analog and digital input/output modules and a Labview program which is used along with a multitrigger digital delaty generator to control various solenoid valves, MKS Baratron transducers, PCB sensors, lasers, and cameras.

Over seventy side-wall ports and plugs are located along the tube to provide access for instrumentation, such as pressure transducers, and optical access for non-intrusive laser diagnostics measurements. To measure the speed of the primary and secondary shockwaves, eight PCB Piezotronics 113B27 pressure sensors are used. Four of the sensors are mounted into ports spaced $30.5 \mathrm{~cm}$ apart located just upstream of the secondary diaphragm, with a similar set up just upstream of the test section. The shock speeds $U_{s 1}$ and $U_{s 2}$ are computed from the time of flight between sensors, and are then used to determine the test gas conditions using a solver which models expansion tubes and includes equilibrium temperature-dependent properties.

Before operation each section of the tube is evacuated with its own designated vacuum pump. During the fill and evacuation process, the pressures in the driven and expansion sections are monitored using Baratron 627D temperature regulated capacitance manometers. These sensors have a resolution of $20 \mathrm{mtorr}$, and were zeroed and are regularly monitored using thermocouple gauge tubes.

\section{Facility Characterization}

\section{A. Selection of Flow Conditions}

Initially, a total of three conditions were designed to be characterized. The conditions were chosen to span a range of properties accessible by the facility while maintaining relevance to the study of supersonic combustion. The first two are referred to as conditions A and B and correspond to Mach 6 and 8 flight, respectively. These flows have aerotherodynamic properties similar to the combustor entry conditions of the hypersonic test-vehicle in the recent HIFiRE Flight-2. During the test window of this experiment, the flight envelope consisted of flight Mach numbers ranging between 6 and 8.5 at a constant dynamic pressure of $86.2 \mathrm{kPa}$. Using this information, a procedure outlined in [11] was followed to estimate the resulting combustor entry properties. The combustor entry pressure as a function of flight altitude for this flight envelope is illustrated in Fig. 4.

There are a number of considerations to be made when designing flow conditions for supersonic propulsion systems. The altitude and Mach number at which these vehicles fly should lie within a certain bounds governed by the dynamic pressure experienced by the aircraft as well as the achievable mass flow rate of oxidizer into the engine. The dynamic pressure defined as, $q=1 / 2 \rho U^{2}$, should generally be taken between the values of 50-100 kPa, as to generate sufficient lift while maintaining acceptable structural loads on the vehicle [11]. As a result, for a hypersonic aircraft to increase its flight Mach number it must fly at higher altitudes, and is generally confined to altitudes of $20-40 \mathrm{~km}$.

The objective of a supersonic inlet is to decelerate the air to a speed sufficient for combustion. This includes increasing the residence time through the combustor to efficiently mix the fuel and oxidizer streams allowing enough time for ignition, and achieving stable flame holding. The compression from the inlet results in an increase in the air's pressure and temperature and can be modeled as an adiabatic process that occurs through a complex shock system before the combustor. Generally, the air entering the combustor should be greater than $1000 \mathrm{~K}$ but should not exceed $1400 \mathrm{~K}$ in order to reduce the amount of radicals produced in the exhaust and give way to a more complete and therefore efficient combustion process [11]. This confines the inlet's adiabatic compression ratio $T_{c} / T_{f}$ to values ranging between 4 and 7, where the subscripts "c" and "f" correspond to combustor and freestream, respectively. By approximating the stagnation temperature $T_{0}$ constant between the freestream and the combustor, the adiabatic 


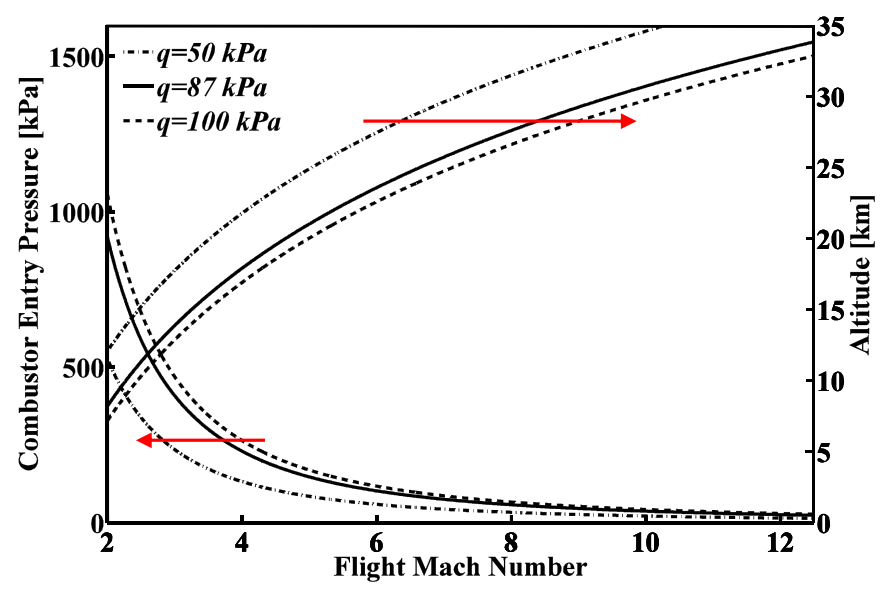

Figure 4. Representative HIFiRE II flight conditions for a flight envelope with a constant dynamic pressure of $87 \mathrm{kPa}$.

compression ratio can be written as such:

$$
\frac{T_{c}}{T_{f}}=\frac{1+\left[\left(\gamma_{f}-1\right) / 2\right] M_{f}^{2}}{1+\left[\left(\gamma_{c}-1\right) / 2\right] M_{c}^{2}}
$$

where $M_{f}$ is the freestream Mach number, $M_{c}$ is the combustor entry Mach number, $\gamma_{f}$ and $\gamma_{c}$ are the ratio of specific heats of the freestream and combustor, respectively. When parameters such as the dynamic pressure and adiabatic compression ratio are fixed, one can estimate the combustor's entry pressure by the following equation:

$$
\frac{P_{c}}{P_{f}}=\left(\frac{T_{c} / T_{f}}{T_{c} / T_{f}\left(1-\eta_{c}\right)+\eta_{c}}\right)^{\left(\gamma_{c} /\left(\gamma_{c}-1\right)\right)}
$$

where $\eta_{c}$ is the compression efficiency of the inlet, $P_{c}$ is the combustor entry pressure, and $P_{f}$ is the freestream pressure. For the design of flow conditions $\mathrm{A}$ and $\mathrm{B}$, the dynamic pressure, adiabatic compression ratio, and inlet compression efficiency were taken to be $87 \mathrm{kPa}, 5$, and 0.70 , respectively.

The third condition referred to as $\mathrm{C}$, is a relatively lower enthalpy flow with a longer test time. It is of interest to the studies of processes that do not require high static temperatures, such as mixing studies. The longer test time was achieved by increasing the ratio of sound speeds $a_{1} / a_{4}$ between the driver and driven sections, relative to those of conditions A and B. This generates a relatively weaker set of incident waves and results in a lower induced velocity of the first contact surface. The second set of expansion waves have a longer time before they are reflected off of the first contact surface and terminate the test time.

\section{B. Summary of Flow Conditions}

An analysis of the resulting experimental flow properties is summarized in Table 1. The uncertainties are standard deviations presented as percentages of the corresponding property's mean value. The uncertainties corresponding to the measured shock speeds as well as semi-theoretical quantities pertain to shot-to-shot variations, and are not related to spatial and temporal uncertainties in the measurement method. For measured aerothermal properties in region 5 of Fig.1, the reported uncertainties are the temporal standard deviations computed over the useful test time. The statistics are based on a total of 15 runs for each condition where the only exception is the measured Mach number, which was analyzed over a fewer number of runs. The aerothermal properties of the test gas are a function of three parameters being the initial fill compositions, initial fill pressures, and shock speeds of the primary and secondary shock waves. Therefore properties labeled as semi-theoretical, were computed based off of these three parameters using an expansion tube gas solver. On the other hand, properties labeled as measured, were in some form directly measured in the experiments. The theoretical test time is based on an inviscid expansion tube solver, which implements the method of characteristics based on the initial fill parameters and lengths of each section and accounts for non-simple regions.

The static pressure measured through the tube wall corresponds to a combined temporal average over the last four shock counters in the expansion section. The average is over a period of time just after the passage of the secondary 


\begin{tabular}{|c|c|c|c|}
\hline Condition & $\mathrm{A}$ & $\mathrm{B}$ & $\mathrm{C}$ \\
\hline \multicolumn{4}{|l|}{ Initial Setup } \\
\hline Composition $\{$ Driver, Driven Expansion $\}$ & $\{\mathrm{He}, \mathrm{Air}, \mathrm{He}\}$ & $\{\mathrm{He}, \mathrm{Air}, \mathrm{He}\}$ & $\{$ Air, $\mathrm{Ar}, \mathrm{He}\}$ \\
\hline Driver Fill Pressure $[\mathrm{kPa}]$ & 1995 & 1825 & 670 \\
\hline Driven Fill Pressure [torr] & 74.3 & 45.4 & 82.8 \\
\hline Expansion Fill Pressure [torr] & 148.2 & 36.6 & 50.2 \\
\hline Nominal Flight Mach Number & 6 & 8 & $\mathrm{NA}$ \\
\hline Primary Shock Speed $[\mathrm{m} / \mathrm{s}] *$ & $1351.5 \pm 0.5 \%$ & $1442.5 \pm 0.6 \%$ & $696.8 \pm 0.3 \%$ \\
\hline Secondary Shock Speed $[\mathrm{m} / \mathrm{s}] *$ & $2160.5 \pm 0.2 \%$ & $2585.0 \pm 0.5 \%$ & $1562.5 \pm 0.3 \%$ \\
\hline Mach Number * & $2.14 \pm 0.1 \%$ & $2.72 \pm 0.9 \%$ & $1.73 \pm 3.3 \%$ \\
\hline Mach Number $\ddagger$ & $2.05 \pm 0.5 \%$ & $2.76 \pm 0.6 \%$ & $1.76 \pm 0.6 \%$ \\
\hline Static Pressure (Test Section) $[\mathrm{kPa}] *$ & $127.3 \pm 0.4 \%$ & $54.4 \pm 0.9 \%$ & $25.6 \pm 0.5 \%$ \\
\hline Static Pressure (Tube Wall) $[\mathrm{kPa}] *$ & $107.6 \pm 0.4 \%$ & $39.1 \pm 0.8 \%$ & $18.3 \pm 0.6 \%$ \\
\hline Static Pressure $[\mathrm{kPa}] \ddagger$ & $106.1 \pm 0.4 \%$ & $38.0 \pm 0.9 \%$ & $18.0 \pm 0.7 \%$ \\
\hline Test Time $($ Total $)[\mu \mathrm{s}] *$ & $949 \pm 5.0 \%$ & $917 \pm 8.8 \%$ & $5540 \pm 1.8 \%$ \\
\hline Test Time (Useful) $[\mu \mathrm{s}] *$ & $550 \pm 7.9 \%$ & $690 \pm 19 \%$ & $4570 \pm 13 \%$ \\
\hline Test Time $[\mu \mathrm{s}] \diamond$ & 1200 & 920 & 4000 \\
\hline Stagnation Enthalpy $[\mathrm{MJ} / \mathrm{kg}] \ddagger$ & $1.83 \pm 0.3 \%$ & $2.3 \pm 0.6 \%$ & $0.30 \pm 0.7 \%$ \\
\hline Static Temperature $[\mathrm{K}] \ddagger$ & $980 \pm 0.5 \%$ & $910 \pm 0.7 \%$ & $420 \pm 0.3 \%$ \\
\hline Velocity $[\mathrm{m} / \mathrm{s}] \ddagger$ & $1259 \pm 0.3 \%$ & $1637 \pm 0.7 \%$ & $673 \pm 0.7 \%$ \\
\hline
\end{tabular}

Table 1. Summary of the MHExT performance. Aerothermodynamic properties pertain to the test gas (region 5).

shock until a time in which the flow is unadulterated by the effects of boundary layer growth. The static pressure measured in the test section is a temporal average over the useful test time using a pitot-static probe assembly which will be described in more detail in the subsequent section.

There are a number of conclusions that can be drawn from the information provided in Table 1. First, the variability in the measured shock speeds from shot-to-shot were measured to vary by a fraction of a percent. This is a result of a combination of reasons being: accurately filling each section of the facility with the desired composition and pressure before each run, having control over when an experiment is initiated, and rupturing the primary and secondary diaphragms in the most ideal manner such that minimal disturbances are introduced to the flow. The consequences of such small variability in the measured shock speeds are shown by the properties labeled semi-theoretical, which were found by carrying out a propagation analysis based on the measured shock speeds.

Secondly, with the exception of test time, the measured properties such as Mach number compare very well with their corresponding semi-theoretical properties. This is important as it shows that the even though expansion tube flows in practice are inherently limited by short test times, boundary layer growth, and flow disturbances, the properties were still measured with very small deviations from the expansion tube solver which does not consider such non-idealities, therefore owing to the facility's ability to generate useful flows.

Third, the measured total test time and theoretical test times vary quite significantly for cases A and C. It is postulated that the difference primarily results from viscous effects such as boundary layer growth. As was investigated by Mirels $[12,13]$, until the limiting separation distance is reached between the contact surface and shock wave, the effect of the boundary layer's growth is to act as an aerodynamic sink and absorb the mass from the core flow. As a result, the shock's speed is attenuated while the contact surface is accelerated. This behavior which is governed by viscous effects, can have a substantial effect on the actual test time of the flow, as was observed in this study.

\section{Flow Mach Number Measurements}

Under ideal steady conditions, axial (along the tube's length) variations in flow properties can be measured temporally as the test gas convects into the test section. In reality, until the limiting distance between the shock and contact surface is reached, the effect of boundary layer growth is a function of both axial distance and time. As a result, measurements taking place in the test section during the test gas flow, are affected by both time and axial position within the test gas slug. In order to understand how these coupled effects alter the test gas properties, pitot and static pressure measurements were carried out simultaneously to directly measure the test gas Mach number.

An assembly (schematic provided in Fig. 5a) which consisted of a flate plate with a leading edge was instrumented with two fast response piezoelectric pressure transducers (PCB 113B21) and was fixed just downstream of the tube's 


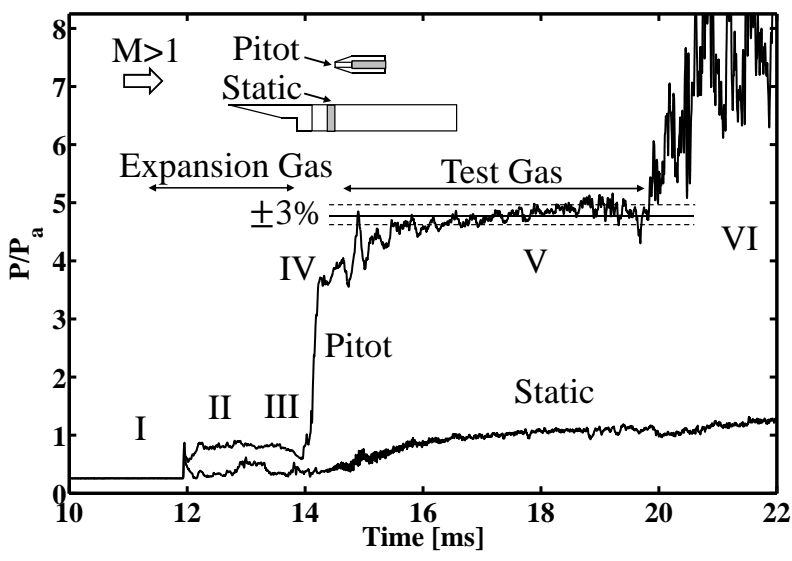

a)

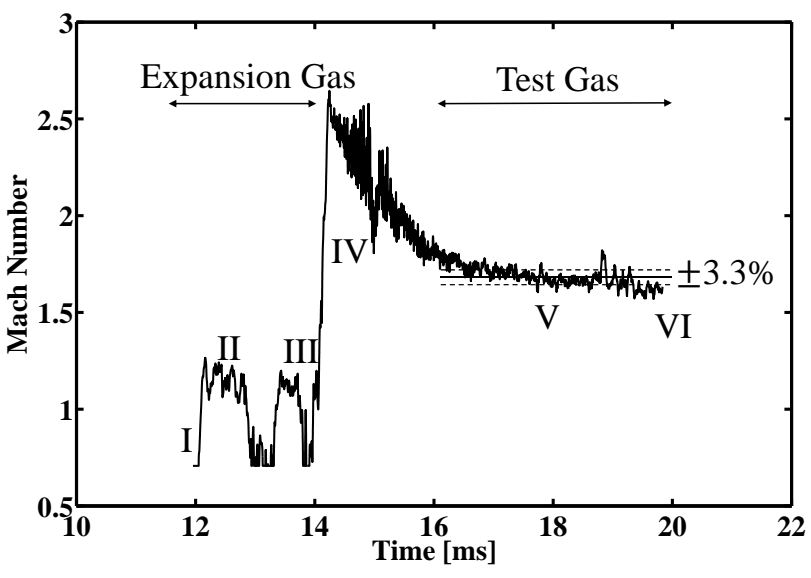

b)

Figure 5. (a) Time-history of simultaneous pitot-static pressure measurements. $P_{a}$ is the mean static pressure over the test time and has a value of $25.6 \mathrm{kPa}$. (b) Time-history of the approximate Mach number inferred from the pitot-static pressure measurements of (a). Data refers to condition $\mathrm{C}$.

exit plane. The static pressure measurements were taken by a flush-mount sensor located approximately 72 mm downstream of the tube's exit plane. The pitot pressure measurements were taken by a sensor which was recessed mounted into a cavity of a pitot tube. The measurements were taken at the same distance downstream of the tube's exit where the pitot sensor was aligned with the center line of the tube approximately 2.5 probe diameters away from the flate plate. The sensor's measurements are limited both spatially and temporally due to: the effective surface area of the sensing element, the inherent response time associated with each sensor, and the response time associated with the cavity of a recessed mounted sensor.

An example of a simultaneous pitot-static pressure measurement for flow condition $\mathrm{C}$ is shown in Fig. 5a. The location labeled I, shows an increase in both static and pitot pressures, and corresponds to the arrival of the secondary shock. This value is equal to the measured tube wall static pressure tabulated in Table 1 and can be seen to match very well with the semi-theoretical calculated value. In order to satisfy kinematic constraints, pressures in regions 20 and 5 are equal and therefore even though this is not the pressure sensed during the presence of the test gas- it corresponds to the test gas's pressure with the presence of minimal boundary layer effects.

The location labeled II corresponds to the flow of the expansion gas over the sensors. Since the Mach number of region 20 is nearly sonic before exiting the tube, the gas behaves as an underexpanded jet as it enters the test section. The mach angle given by $\mu=\sin ^{-1}\left(\frac{1}{M}\right)$ will have a large value resulting in an expansion cone that does not envelop the static sensor. Therefore, the decrease of static pressure in location II compared to I is due to the pressure sensed downstream of the steadily expanded expansion gas.

Since the Mach number of the expansion and test gas are different, the pitot traces allow us to more accurately discern the different regions of the flow. The location labeled III indicates the arrival of the supersonic test gas and hence an increase in the measured pitot pressure. An increase in the static pressure is also observed resulting from the change in mach angle of the underexpanded jet as the expansion cone begins to envelop the sensor. The location of IV corresponds to a period of non-uniform flow over the sensors. During this time, we observe the transient processes associated with the change in the resulting wave structure as the test gas begins to exit the tube. The pitot trace in region IV is an artifact of this transient process as well as the delayed response time associated with the recessed mounted sensor in the pitot tube.

Following the transient flow of region IV is the uniform test gas flow labeled as V. This is the region of useful test time and it is terminated (label VI) by the arrival of any of the rarefaction waves. The measured property 'total test time' in Table 1, refers to the time between the arrival of the test gas (III) and the arrival of any of the rarefaction waves (VI). This is the ideal length of the test time if the contact surface were truly discontinuous and if viscous effects were negligible. On the other hand, the measured property 'useful test time' refers to region $\mathrm{V}$ and corresponds to the region of the flow in which the pitot trace does not exceed $\pm 5 \%$ of the average value.

The property labeled 'static pressure (test section)' in Table 1, is the average measured static pressure during the useful test time (V) obtained from the static sensor in the test section. The value of this pressure is always greater than what is measured when the secondary shock first arrives (I), at the wall of the tube immediately after the secondary 


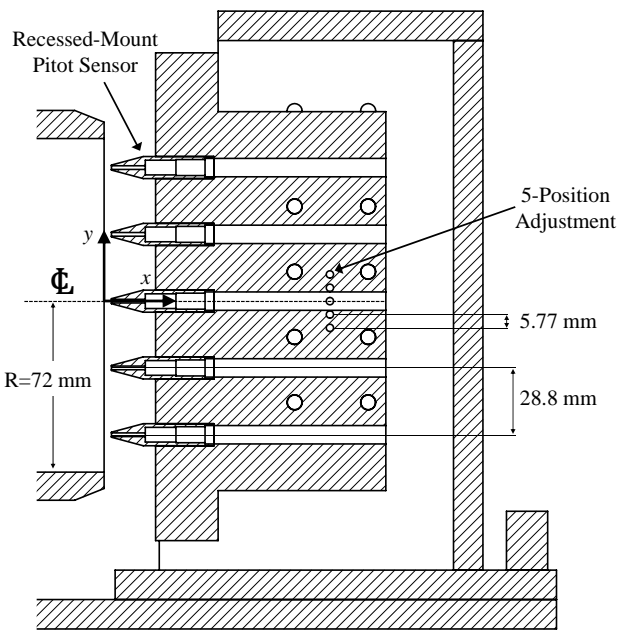

Figure 6. Schematic of pitot rack assembly in the test section. In this configuration the rack is in its radially centered position.

shock wave, or compared to the semi-theoretical calculation. This is a result of boundary layer growth in the expansion tube which reduces the effective core flow diameter, and in turn compresses the test gas. Since the test gas travels a considerable distance before reaching the test section, the growth of the boundary layer will have caused non-uniform, axial variations in the test gas properties. These are sensed as temporal variations in pressure with the pitot and static sensors.

The Mach number of the test gas was calculated using the Rayleigh pitot tube formula from the measured pitot and static pressure time traces (using an average value of $\gamma$ between the pre- and post-normal-shock values). Figure $5 \mathrm{~b}$ shows an approximate time-history of the flow Mach number as inferred by the simultaneous pitot-static pressure measurements. Although the values of pressure used to calculate the test gas Mach number in region $\mathrm{V}$ are greater than the ideal inviscid values, the Mach number during V compares very well with the semi-theoretical calculation. Therefore, the increase in static pressure is off-set by an increase in pitot pressure which results in a flow with a nearly constant Mach number matching the value predicted by the inviscid calculations.

\section{Radial Measurements}

One of the inherent limitations of using expansion tubes to generate useful gas flows is due to the adverse effects of the boundary layer's growth. Since the test gas must travel several tube diameters downstream until it reaches the test section, the growth of the boundary layer may have a significant effect on the radial uniformity of the flow properties and the size of the core flow. Therefore, it is important to have an understanding of what the effective core size of the flow is and how test gas properties vary along the tube's diameter during the useful test time.

Spatial pressure measurements were carried out using a pitot rack shown in Fig. 6, which was fixed just downstream of the tube exit. The rack was originally designed by Orley [14] to measure a total of 25 evenly distributed positions spanning the diameter of the tube $(2 R=144 \mathrm{~mm})$. This was done by instrumenting the rack with 5 pitot tubes evenly spaced $28.8 \mathrm{~mm}$ apart. The rack was then traversed and locked through a total of 5 positions evenly spaced $5.8 \mathrm{~mm}$ apart. Each canonical pitot tube housed a fast-response piezoelectric pressure sensor which was recessed mounted into the cavity of the tube. Unfortunately, due to calibration issues with one of the sensors some portion of data was omitted from the present work and therefore, the pitot pressure profiles consist of 20 measurement points. A minimum of two experiments were done for each pitot rack position.

For each experimental run, pitot pressure measurements were temporally averaged over the useful test time. A sample of time-history pitot pressure measurements from four radial locations across the diameter of the tube for Condition A is provided in Fig. 7a. The roman numerals correspond to the description outlined in the previous section. The pitot measurements taken at positions $r / R=0, r / R=0.4$, and $r / R=-0.4$ are shown to all lie within the core flow. The traces not only have the same average value over the test time, but they also qualitatively have the same trending behavior indicating that the flow is uniform in the radial direction. The pitot probe in position $r / R=0.8$ exemplifies a trace that is in the boundary layer of the flow. This can be seen by the relatively lower mean pitot pressure (lower Mach number) and larger flow fluctuations.

The radial profile of the average pitot pressure for condition A is shown in Fig. 7b. The $x$-axis of the figure is 


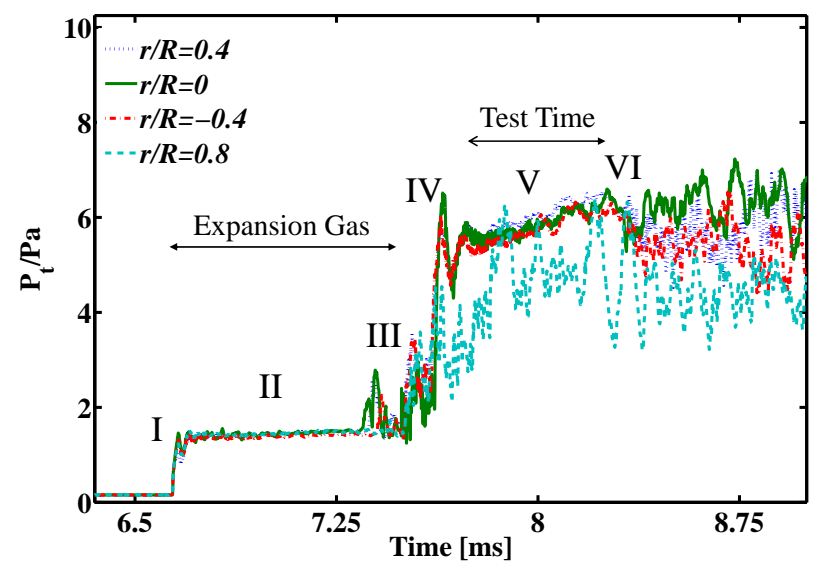

a)

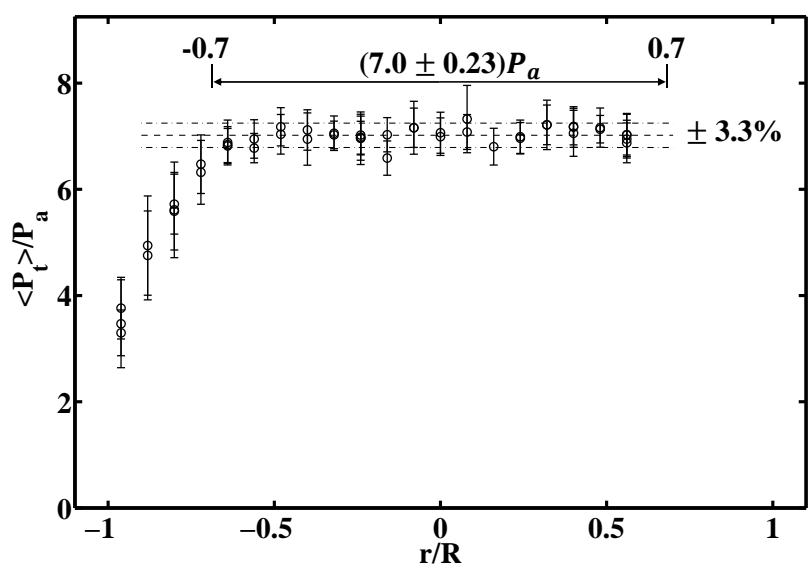

b)

Figure 7. (a) Time-history of pitot pressure measurements at three radial locations along the tube diameter for Condition A. (b) Radial profile of the mean pitot pressure measurements for Condition $\mathrm{A}$ taken just downstream of the tube exit to asses test gas flow uniformity in both the radial direction. $P_{a}$ is the mean static pressure over the test time and has a value of $127.3 \mathrm{kPa}$. Data refers to Condition A.

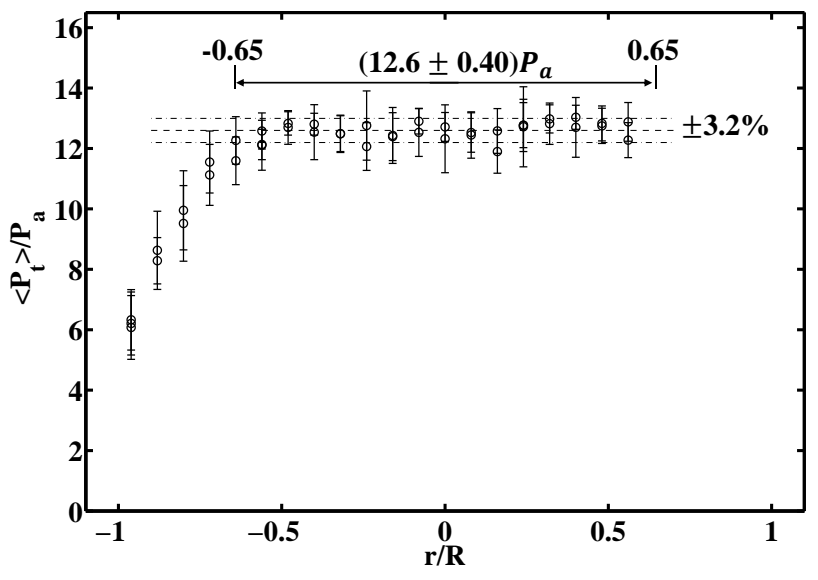

a)

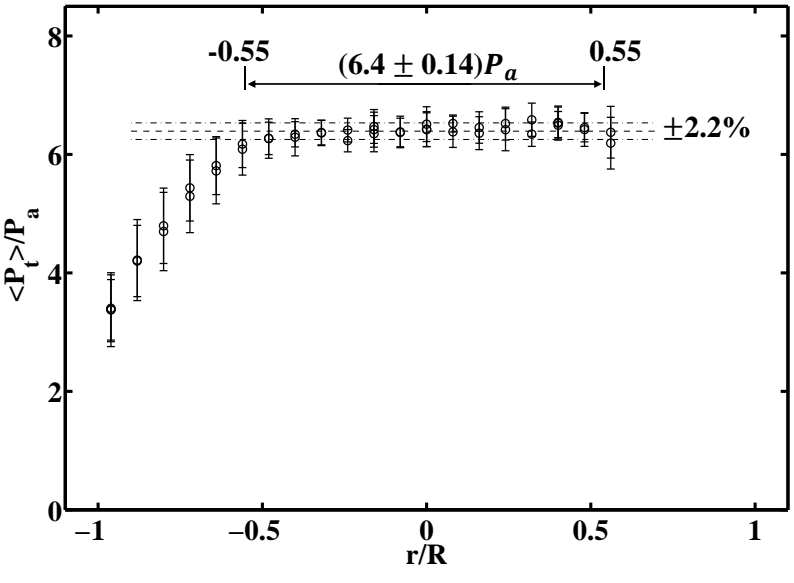

b)

Figure 8. Radial profile of the pitot pressure measurements for (a) Condition $B$ and (b) Condition $C$ taken just downstream of the tube exit. $P_{a}$ is the mean static pressure over the test time and has a value of $54.4 \mathrm{kPa}$ and $25.6 \mathrm{kPa}$ for conditions $\mathrm{B}$ and $\mathrm{C}$, respectively.

normalized by the inner radius of the tube, and the $y$-axis was normalized by an average measured static pressure value of $127.3 \mathrm{kPa}$ from Table 1. The error bars correspond to the standard deviation measured over the useful test time. The core flow was established to span $70 \%$ of the inner diameter of the tube. The standard deviation in the core region was found to be within $\pm 3.3 \%$ of the mean value, which is equal to $7.0 P_{a}$. A similar procedure was followed for flow conditions $\mathrm{B}$ and $\mathrm{C}$ and are provided in Fig. 8. The results show that for all three cases, the flow properties across the tube diameter remain to within $\pm 5 \%$ of the mean value in the core flow, which spans approximately $3 / 4$ th of the tube diameter. There is, however, a small variation of the effective core size varies among the cases; the core size increases with increasing test gas pressure.

\section{E. Schlieren Imaging}

A standard Z-type schlieren system was set up to acquire $\mathrm{kHz}$ frame-rate schlieren images of the generated flow field as it developed over a $20^{\circ}$ wedge. The high-speed images were captured at a frame rate of $24 \mathrm{kHz}$ with an exposure of $2 \mu \mathrm{s}$. The illumination was provided by an LED (Luminus SBR-70), which was collimated through the test section with a combination of lenses and spherical mirrors. The knife-edge was oriented horizontally to visualize density gradients in the vertical direction. Static pressure measurements downstream of the oblique shock were simultaneously 


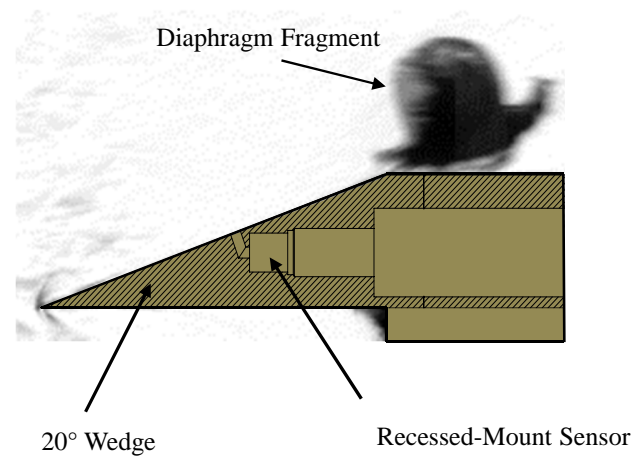

a)

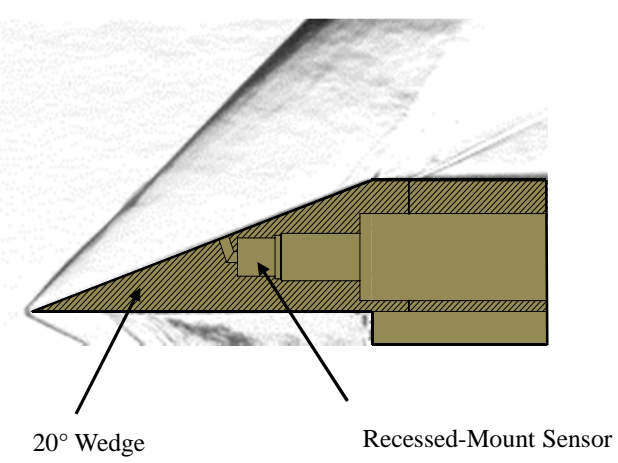

c)

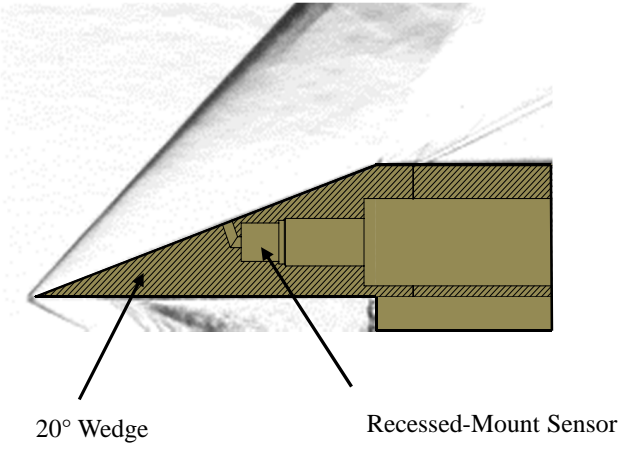

b)

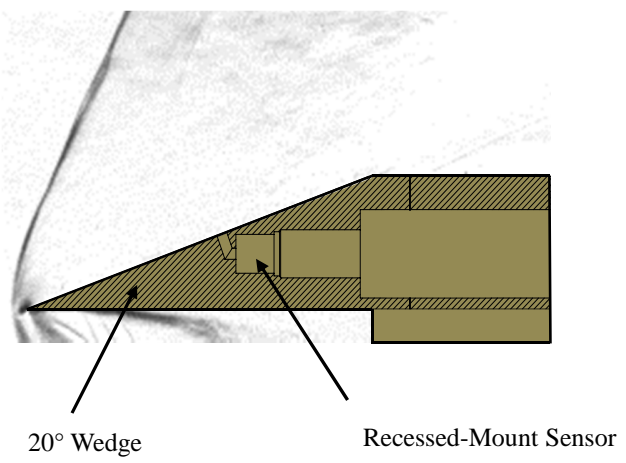

d)

Figure 9. Time sequence of the flow structure around the $20^{\circ}$ wedge instrumented with a fast-response recessed-mounted static sensor. The sequence is extracted from the high-speed $(24 \mathrm{kHz})$ schlieren movie: (a) flow soon after arrival of the secondary shock wave (I); (b) flow during the time the supersonic expansion gas is flowing over the wedge (II); (c) flow during the useful test time (V); and (d) flow soon after the termination of the useful test time (VI). Data refers to Condition A.

acquired through a recessed-mounted fast response piezoelectric pressure transducer instrumented inside the wedge. The captured images were reduced by removing dark noise and normalizing by the mean illumination field.

Examples of a few still images extracted from the schlieren movie at different instances during the evolution of the flow are shown in Fig. 9. The sequence refers to flow condition A. The set of images shows the schlieren images overlaid on a schematic of the wedge showing the position of the sensor inside of the wedge. The first image, Fig. 9a, shows the flow field several microseconds after the arrival of the secondary shock (I). The supersonic helium flow is just beginning to flow over the wedge and to form a shock wave. The second image, Fig. 9b, shows the oblique shock generated during the flow of the supersonic expansion gas over the wedge. The measured wave angle $\beta=48.0^{\circ}$ corresponds to an expansion gas flow Mach number of 2.45. Figure 9c shows the flowfield at one instant during the useful test time (V). Finally, Fig. 9d shows the flow after the test time flow terminates (VI): in this case the flow Mach number decreases and as a result, the oblique shock detaches. Figure 10a shows the corresponding time-history of the wall static pressure measured on the surface of the wedge behind the oblique shock; labels identify the main phases of the flow evolution described previously.

From the set of schlieren images, an average Mach number of the flow upstream of the oblique shock $M_{5}$, was computed using the $M-\beta-\theta$ relation (for constant $\gamma$ ) from [15]. Here we used a value of $\gamma$ equal to the average between the values across the oblique shock. The measured oblique shock angle during the useful test time was $53.2^{\circ}$ which corresponds to a value of $M_{5}=1.96$. The uncertainty in measuring the wave angle translates to an uncertainty of $\pm 1.5 \%$ on $M_{5}$. This measured value of $M_{5}$ is $4.3 \%$ lower than the computed semi-theoretical value of 2.05 . The measured time-averaged static pressure on the surface of the wedge during the useful test test was $317 \mathrm{kPa}$ which corresponds to a pre-shocked static pressure of $118 \mathrm{kPa}$. This value is $7.9 \%$ lower than the measured value using the pitot-static probe assembly. Fig.10b shows a time-history of the inferred flow Mach number upstream of the oblique shock $M_{5}$. The vertical and horizontal error bars correspond to uncertainties in measuring the oblique shock angle and temporally correlating the images to the pressure trace, respectively. The results show a region of uniform test gas flow where the standard deviation of the Mach number during the useful test time (V) is $0.7 \%$ of the mean value. 


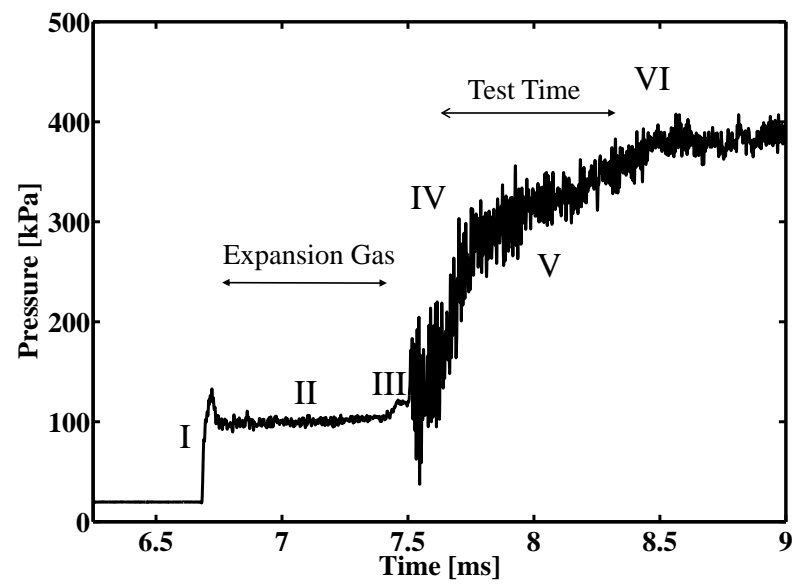

a)

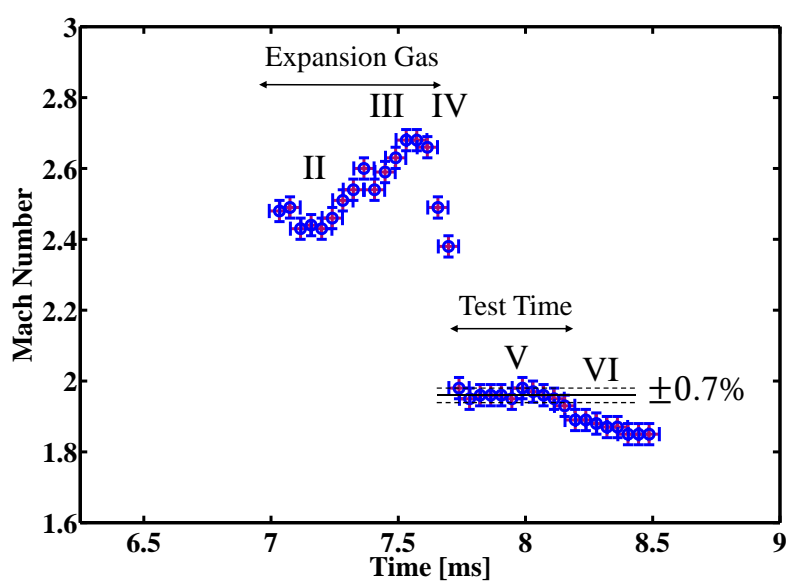

b)

Figure 10. (a)Time-history of wall static pressure measured on the wedge surface behind the oblique shock. (b)Inferred gas Mach number based on measured oblique shock angle. Data refers to Condition A.

\section{Improvements in Pressure Measurement Capabilities}

The use of fast-response piezoelectric pressure sensors are the favored method for dynamic pressure measurements with impulse facilities such as expansion tubes. This is due to their ability to survive in harsh environments and respond within micro-seconds to step changes in pressure, while providing high SNR (signal-to-noise ratio) measurements. A series of experiments using the simultaneous pitot-static pressure measurement assembly (see Fig. 11a for a schematic view) revealed a shortcoming in the ability of the sensor to accurately measure static pressure in such environments. Upon arrival of the test gas, the voltage output from the sensor is overwhelmed by high amplitude noise which renders the pressure measurement useless. Since the sensors are not intended to measure radial loadings of pressure onto the sensing element and only designed and calibrated to measure forces acting axially along the sensor's length, the vibrations induced by the high stagnation pressures of the test gas give way to measurements that are of no use.

An example of a pressure trace with overwhelming amounts of noise is shown by the red pressure trace in Fig. $11 \mathrm{~b}$. This pressure trace was taken for flow condition B, and is a typical example of any static pressure trace acquired with the original mounting configuration of the sensor. This configuration consisted of flush-mounting the sensor, in a manner specified by the manufacturer, directly into the ground polished aluminum plate. Upon arrival of the test gas (III), the signal clearly failed to provide usable data as the pressure trace registered a negative value of pressure, and a standard deviation of $P_{\text {std }}=56 \mathrm{kPa}$.

An alternative method of mounting the static sensor based on the work of Ref. [10] was proposed and tested. The modified design is shown schematically in Fig. 11a. With this configuration, the static sensor is flush-mounted into a delrin sleeve with a diameter approximately equal to 3 sensor body diameters, in a manner specified by the manufacturer. The aluminum plate was bored with a diameter $2 \mathrm{~mm}$ larger than that of the delrin sleeve. The sleeve was flush mounted into the plate while high-temperature RTV silicone was used to fill the space between the parts. The motivation behind this design was to provide a way for the sub-assembly holding the sensor to dampen the vibrations induced by the strong pressure waves resulting from the arrival of the test gas. Thus, the combination of delrin and RTV silicone were chosen to independently and collectively dissipate the mechanical energy in the form of vibrations by acting as a visco-elastic dampener. The use of delrin is also suitable for machining purposes and electrically insulates the sensor from the rest of the assembly. In addition, the pressure sensor was also grounded. This was done by attaching a ground strap from the sensor to the inside wall of the test section. This provided a pathway for electrically induced noise to move through, instead of contaminating the signal going to the signal conditioner. The electrically induced noise may be a result of vibrations of the electrical cables, as well as the inherently high temperatures of the flow field. An example of the static pressure trace with the modified configuration is shown by the green line in Fig.11b. As a result of this alternative mounting strategy, there is a significant improvement in both the qualitative and quantitative features of the signal. The static pressure measurements reported here were acquired with this sensor mounting configuration. 


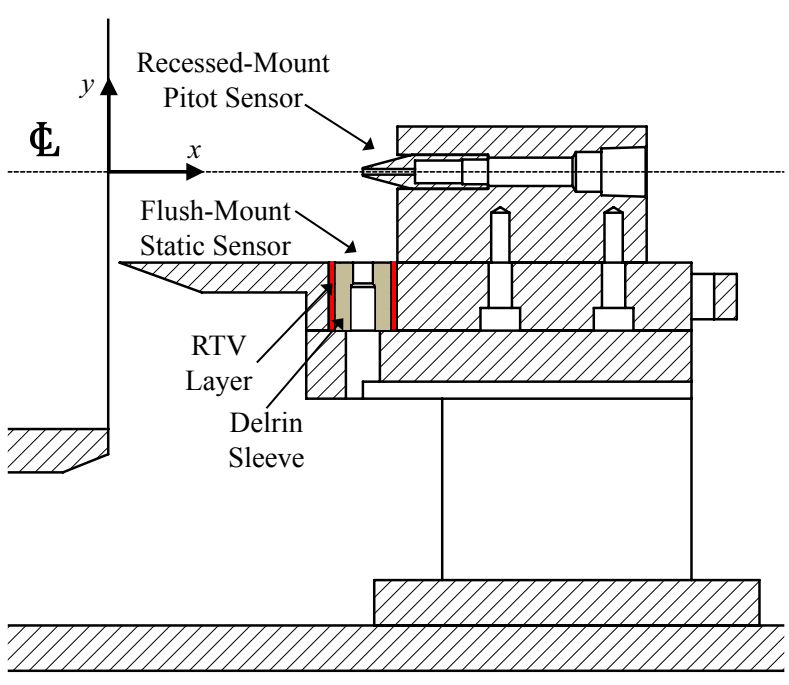

a)

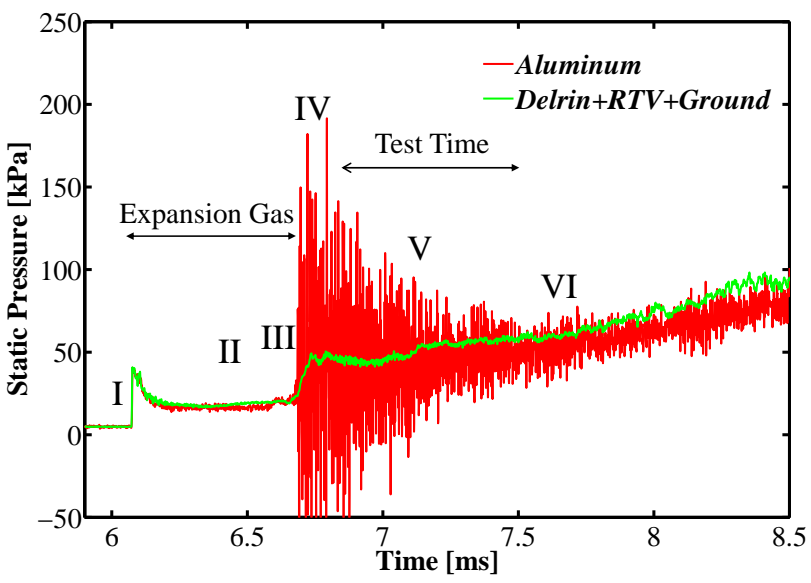

b) Condition B

Figure 11. (a) Schematic of simultaneous pitot-static pressure measurement assembly in the test section with the modified static sensor mounting. (b) Quantitative time-history comparison of original and modified static sensor measurements for flow condition B, taken during two separate experiments.

\section{Conclusions}

A new hypersonic impulse facility has been designed and constructed in the Gas Dynamics Imaging Lab at the University of Michigan. The facility was deigned to generate aerothermal flow conditions for flight Mach numbers ranging between 4 and 11. A number of key design considerations are discussed along with an overview of the operation of the facility. The capabilities of the facility were assessed experimentally by evaluating the ability to repeatably, accurately, and uniformly generate flow conditions of interest. Three flow conditions spanning a range of aerothermal flow properties, while maintaining relevance to the studies of supersonic combustion were designed and used for this study.

A shot-to-shot statistical analysis of the measured shock speeds reveal a variability amounting to a fraction of a percent for all three conditions. The measured static pressure of the test gas at the tube wall, before being altered by boundary layer effects, agree well with semi-theoretical values. The extent to which the boundary layer affects the test gas properties was evaluated using a number of assemblies and experimental methods. The useful test time, a region in which the pressure traces do not exceed $\pm 5 \%$ of their mean value, was established for each condition.

The results of simultaneous pitot-static pressure measurements reveal a strong agreement between the measured temporal average and semi-theoretical value of test gas Mach number, in spite of the increase in both pitot and static pressure over the length of the test gas slug as it convects into the test section. Simultaneous kHz frame-rate schlieren imaging of the flow field as it developed over a $20^{\circ}$ wedge, and static pressure measurements on the wedge surface were carried out. The acquired images were temporally correlated with their corresponding pressure trace and the oblique shock angle in the images were measured to infer the generated gas Mach number as a function of time. The results show a region of test gas with a uniform Mach number convecting over the wedge.

Spatial pressure measurements with a pitot rack were used to characterize the core flow size and spatial uniformity of the test gas flow. A total of 20 radial locations along the expansion tube's exit plane were used to construct radial pressure profiles of the test gas flow. The core flow size varied between $r / R=0.55$ and $r / R=0.70$ among all three cases, where the core size was larger with higher values of test gas pressure. The temporal mean value of the core flow pressure during the useful test gas at each radial location was found to vary by less than $\pm 3.3 \%$ of the mean core pressure.

\section{References}

[1] Jackson, K. R., Gruber, M. R., and Buccellato, S., "Mach 6-8+ Hydrocarbon-Fueled Scramjet Flight Experiment: The HIFiRE Flight 2 Project," Journal of Propulsion and Power, July 2014, pp. 1-18.

[2] Smart, M. K., Hass, N. E., and Paull, A., "Flight Data Analysis of the HyShot 2 Scramjet Flight Experiment," AIAA Journal, Vol. 44, No. 10, Oct. 2006, pp. 2366-2375. 
[3] Jackson, K., Gruber, M., and Buccellato, S., "HIFiRE Flight 2 Overview and Status Update 2011," 17th AIAA International Space Planes and Hypersonic Systems and Technologies Conference, , No. April, pp. 1-17.

[4] Dufrene, A., Maclean, M., and Wadhams, T., "Characterization of the New," 48th AIAA Aerospace Sciences Meeting Including the New Horizons Forum and Aerospace Exposition, , No. January, pp. 1-14.

[5] John, I., Bakos, R. J., Castrogiovanni, A., Rogers, R. C., and Rogers, R. C., "Dual mode shock-expansion / reflected-shock tunnel," AIAA, Aerospace Sciences Meeting \& Exhibit, , No. January.

[6] DOOLAN, C., "Design and construction of the X-2 two-stage free piston driven expansion tube," 1995. 18, 1995.

[7] Dufrene, a., Sharma, M., and Austin, J. M., "Design and Characterization of a Hypervelocity Expansion Tube Facility," Journal of Propulsion and Power, Vol. 23, No. 6, Nov. 2007, pp. 1185-1193.

[8] Heltsley, W., Snyder, J., Houle, A., Davidson, D., Mungal, M., and Hanson, R., "Design and Characterization of the Stanford 6 Inch Expansion Tube," 42nd AIAA/ASME/SAE/ASEE Joint Propulsion Conference \& Exhibit 9 - 12 July 2006, Sacramento, California AIAA, , No. July, pp. 1-12.

[9] Trimpi, R., "Preliminary Theoretical Study of the Expansion Tube, A New Device for Producing High Enthalpy Short Duration Hypersonic Gas Flows," NASA Technical Report, 1962.

[10] Miller, V. A., Gamba, M., Mungal, M. G., and Hanson, R. K., "Secondary Diaphragm Thickness Effects and Improved Pressure Measurements in an Expansion Tube,” AIAA Journal, Vol. 52, No. 2, Feb. 2014, pp. 451-456.

[11] Heiser, W. H. and Pratt, D. T., Hypersonic Airbreathing Propulsion, American Institute of Aeronautics and Astronautics, sixth ed., 1994.

[12] Mirels, H., "Shock tube test time limitation due to turbulentwall boundary layer," AIAA Journal, Vol. 2, No. 1, 1964 , pp. 1-10.

[13] Mirels, H., "Flow Nonuniformity in Shock Tubes Operating at Maximum Test Times," Physics of Fluids, Vol. 9, No. 10, 1966, pp. 1907.

[14] Orley, F., A Study of Expansion Tube Gas Flow Conditions for Scramjet Combustor Model Testing, Ph.D. thesis, Technische Universitat Munchen, 2011.

[15] Liepmann, H. W. and Roshko, A., Elements of Gas Dynamics, John Wiley and Sons, 1957.

[16] Orley, B. F., Strand, C. L., Miller, V. A., Gamba, M., Adams, N. A., and Iaccarino, G., "A study of expansion tube gas flow conditions for scramjet combustor model testing," Center for Turbulence Research Annual Research Briefs 2011, 2011, pp. 285-296. 